\title{
PERFORMANCE ANALYSIS OF AN ISMA CDMA PACKET DATA NETWORK
}

\author{
Jordi Pérez-Romero, Ramón Agusti, Oriol Sallent \\ Department of Signal Theory and Communications - Polytechnic University of Catalonia (UPC) \\ C/ Jordi Girona, 1-3, Campus Nord-Edifici D4 08034 Barcelona-Spain \\ e-mail: [jorperez, ramon, oriol]@tsc.upc.es
}

\begin{abstract}
Efficiency of MAC protocols for a packet switching DS-CDMA network strongly depends on the offered traffic statistic. For bursty sources where transmission needs are restricted to short message lengths, a protocol such as S-ALOHA can be efficient. However, for longer messages, a reduction in the access randomness is required in order to improve the system performance. This improvement can be provided by a protocol such as ISMA when combined with the DS-CDMA technique. In this paper an analytical approach to model the resulting ISMA CDMA scheme is presented, and some design issues regarding the number of codes to be used are presented.
\end{abstract}

\section{1.- Introduction}

In the recent years we are assisting to a worldwide expansion in the demand for different kinds of integrated multimedia services involving bursty traffic sources. Consequently, for its inherent ability to integrate multiple traffic sources, the interest on DS-CDMA packet radio networks is being increased. Under this framework, the research for a MAC (Medium Access Control) protocol which manages efficiently the different resources (i.e. available codes and power allocation) becomes necessary.

In the presence of bursty traffic the assignment of a dedicated code sequence to each user is inefficient, so other alternatives such as the purely random access S-ALOHA, where a user randomly chooses a code from the total number of available codes and transmits a single packet on it, become more attractive under low traffic loads. However, when the number of packets to be transmitted by a single user increases, the efficiency is reduced due to collisions in the code acquisition process that must be performed for each of the packets. In such a case, it is better to reduce the randomness in the access for example by allowing a user that successfully transmits a packet on a certain code to keep it until the end of the current message

This work was supported by CICYT project TIC 98-684. transmission. The base station would then indicate which codes are currently being used and thus the access to those codes would be inhibited. The socalled ISMA (Inhibit Sense Multiple Access) protocol was studied in [1] but not in a DS-CDMA environment, while in [2] an analysis of a protocol with the same fundamentals as ISMA is presented in the context of CDMA. Nevertheless, this analysis doesn't account for the multiuser interference, which originates that even a single packet transmission on a given code can be received with errors and thus a retransmission can be required.

Based on this framework, this paper presents an analytical model for an ISMA CDMA scheme taking into account both the multiuser interference and the collision in the code acquisition process. The remaining of the paper is organized as follows. In section 2, the analytical model is developed, while in section 3 a performance analysis is carried out by determining an optimal number of codes to be used in this protocol. Finally, conclusions are summarized in section 4 .

\section{2.- ISMA CDMA model}

We consider the situation where $N$ registered users share a set of $K$ code sequences. The base station broadcasts the state of the different code sequences in the current time slot (0: free, 1: acquired) and also the result for each packet transmission in the previous time slot ( 0 : incorrect, 1: correct). Each user can be in one of the following operation modes:

Idle: Terminals generate new messages with probability $p_{i}$. The number of $L$-bit packets in each message follows a geometrical distribution with a mean $\left(1 / p_{m}\right)$. Whenever a message is generated, in the next slot the terminal will randomly choose one of the available codes and the first packet will be transmitted on it. If this packet is correctly received and the message still contains other packets, then the terminal goes to transmission mode and the base station will mark the selected code as acquired.

Backlogged: Users enter this state whenever the first packet sent was received in error either due to a collision with another user that chose the same code or to multiuser interference. Users in backlogged mode will randomly select another 
available code and try the transmission again in the next slot with probability $p_{r}$.

Transmission: In this operation mode, terminals just send packets by making use of the previously acquired code until the completion of the message transmission. Whenever the transmitted packet is not correctly received due to multiuser interference, terminals will go to retransmission mode although the code sequence remains reserved (i.e. the base station marks the code as acquired). As soon as the last packet in the message is correctly transmitted, terminals go back to idle mode and the base station will mark the code that was being used as free.

Retransmission: With probability $p_{b}$ users go back to transmission mode in order to retry the transmission of the incorrectly received packet.

Let $N_{k}^{B}, N_{k}^{T}, N_{k}^{R}$ be the number of users in backlogged mode, transmission mode and retransmission mode at the beginning of the $k$-th time slot. It is easy to show that $\left\{N_{k}^{B}, N_{k}^{T}, N_{k}^{R}\right.$ defines a finite state discrete time Markov chain, so we are able to determine the system performance just by finding the steady state probabilities:

$\pi_{i, m, r}=P\left[N^{B}=i, N^{T}=m, N^{R}=r\right]$

Where $0 \leq r \leq K, 0 \leq m \leq K-r, 0 \leq i \leq N-m-r$. It must be noted that the subscript $k$, indicating time dependence, has been removed indicating the steady state situation.

According to the different operation modes, the system behavior is shown in figure 1 for the case when there are available codes at the beginning of the $k$-th time slot. Our objective here is to derive the state transition probabilities.

By applying the law of total probability and the relationships between parameters that are directly deduced from figure 1 we can obtain expression (2) which is valid whenever there are available codes in the present slot $(K-m-r>0)$. In this expression we can distinguish 6 terms which can be calculated directly and which respond to the six different processes that are involved in the protocol:

(a) This term is related with the transmission process. It accounts for the probability that, whenever there are $m$ users in transmission mode and $n$ users who try to acquire a code, there is a certain number of users $N_{k}^{F T}$ that enter the retransmission mode and a certain number of users $N_{k}^{T S}$ that complete the message transmission and return back to idle mode. The calculation of this probability is immediate from the transitions diagram in figure 1 by noticing that a terminal in transmission mode can perform one out of three actions: 1.- it returns back to idle mode with probability $p_{o k}(m+n) p_{m}$, meaning that the current transmission is successful and it corresponds to the last packet in the message; 2.- it moves to retransmission mode with probability (1$\left.p_{o k}(m+n)\right)\left(1-p_{b}\right)$ meaning that the current transmission contains errors and that the retransmission is not to be performed in the next slot; or 3.- in all other cases it remains in transmission mode. Consequently, we have a trinomial distribution with parameters $\left(m, p_{o k}(m+n)\right.$ $\left.p_{m},\left(1-p_{o k}(m+n)\right)\left(1-p_{b}\right)\right)$, and thus the considered factor can be evaluated as:

$$
\begin{aligned}
& P\left[N_{k}^{T S}=c-w-l+m-s+r, N_{k}^{F T}=s-r+t \mid N_{k}^{A}=n, N_{k}^{T}=m\right]= \\
& =\left(\begin{array}{c}
m-s+r-t \\
c-w-l+m-s+r
\end{array}\right)\left(\begin{array}{c}
m \\
s-r+t
\end{array}\right)\left(p_{o k}(m+n) p_{m}\right)^{c-w-l+m-s+r} \\
& \left(1-p_{o k}(m+n) p_{m}-\left(1-p_{o k}(m+n)\right)\left(1-p_{b}\right)\right)^{l-c+w-t} \\
& \left(\left(1-p_{o k}(m+n)\right)\left(1-p_{b}\right)\right)^{s-r+t}
\end{aligned}
$$

where the probability of receiving a correct $L$-bit packet when there are $x$ simultaneous transmissions is given by:

$p_{\text {ok }}(x)=\left(1-p_{e}(x)\right)^{L}$

and the bit error probability for an interference limited DS-CDMA system with $x$ simultaneous users according to the Gaussian approximation [3] with spreading factor $S_{f}$ is given by:

$$
p_{e}(x)=Q\left(\sqrt{\frac{3 S_{f}}{x-1}}\right)
$$

If we assume that there exist several power control periods during the packet transmission time, as it is specified in the UTRA proposal [4], we can consider a perfect power control scheme, and thus expression (5) can be applied.

(b) The second term reflects the number of terminals that go from retransmission mode to transmission mode in a given slot. As this decision is performed with probability $p_{b}$, this number follows a binomial distribution, which gives:

$P\left[N_{k}^{T R}=t \mid N_{k}^{R}=r\right]=\left(\begin{array}{l}r \\ t\end{array}\right) p_{b}^{t}\left(1-p_{b}\right)^{r-t}$

(c) This term accounts for the terminals that although successfully acquiring a code, they don't keep it because they only have a single packet to transmit. It is again a binomial distribution, that can be calculated as:

$P\left[N_{k}^{T 1}=w \mid N_{k}^{S A}=c\right]=\left(\begin{array}{c}c \\ w\end{array}\right) p_{m}^{w}\left(1-p_{m}\right)^{c-w}$

(d) The fourth term reflects the code acquisition process. It accounts for the probability that $c$ users acquire a code successfully when there are $K-m-r$ available codes and $n$ users who choose one of them. Here we must distinguish between the two 
effects than can bring on a failure: these are collisions and interference.

If we firstly focus on collisions, we are interested in the probability $P\left(c_{2}, n, K-m-r\right)$ of having a number of successes $c_{2}$ when $n$ users randomly choose one out of $K-m-r$ codes and considering that a success means that only one user has selected a given code. This probability is given in expressions (8), (9) according to the derivation presented in [5].

Additionally we must take into account the effect of interference, that can originate a packet to be incorrect even if no other user has selected the same code. Then, considering that in a given slot the total number of simultaneous users is $n+m$, the probability that we are looking for is given by:

$$
\begin{aligned}
& P\left[N_{k}^{S A}=c \mid N_{k}^{A}=n, N_{k}^{T}=m, N_{k}^{R}=r\right]= \\
& =\sum_{c_{2}=c}^{m i n(n, K-m-r)}\left(\begin{array}{c}
c_{2} \\
c
\end{array}\right)\left(p_{o k}(m+n)\right)^{c} \\
& \left(1-p_{o k}(m+n)\right)^{c_{2}-c} P\left(c_{2}, n, K-m-r\right)
\end{aligned}
$$

(e) This term accounts for the number of users in the backlogged mode that decide to choose a code. Again, it can be calculated by means of a binomial distribution:

$$
\begin{aligned}
& P\left[N_{k}^{A B}=n-j+i-c \mid N_{k}^{B}=i\right]= \\
& =\left(\begin{array}{c}
i \\
n-j+i-c
\end{array}\right) p_{r}^{n-j+i-c}\left(1-p_{r}\right)^{c-n+j}
\end{aligned}
$$

(f) Finally, the last term accounts for the number of users that leave the idle mode because they start a new message transmission. Similarly to the previous term, we obtain:

$$
\begin{aligned}
& P\left[N_{k}^{A I}=j-i+c \mid N_{k}^{I}=N-i-m-r\right]= \\
& =\left(\begin{array}{c}
N-i-m-r \\
j-i+c
\end{array}\right) p_{i}^{j-i+c}\left(1-p_{i}\right)^{N-m-r-j-c}
\end{aligned}
$$

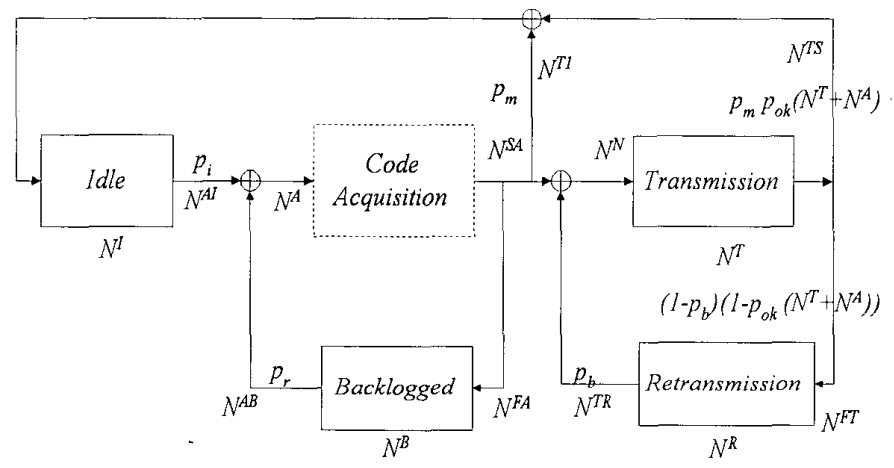

Figure 1 State transitions for the ISMA CDMA model

$$
\begin{aligned}
& p_{i m r, j l s}=P\left[N_{k+1}^{B}=j, N_{k+1}^{T}=l, N_{k+1}^{R}=s \mid N_{k}^{B}=i, N_{k}^{T}=m, N_{k}^{R}=r\right]= \\
& =\sum_{n=0}^{N-m-r \min (K-m-r, n, n-j-j+i, N-m-r-j) \min (c, c-l+m-s+r) \min (r, m-s+r, l-c+w)} \sum_{c=\max (0, i-j, n-j)} \sum_{t=\max (0, r-s)}^{P\left[N_{k}^{T S}=c-w-l+m-s+r, N_{k}^{F T}=s-r+t \mid N_{k}^{A}=n, N_{k}^{T}=m\right]} \\
& \underbrace{P\left[N_{k}^{T R}=t \mid N_{k}^{R}=r\right]}_{(b)} \underbrace{\left[\left[N_{k}^{T 1}=w \mid N_{k}^{S A}=c\right]\right.}_{(d)} \underbrace{\left[\left[N_{k}^{S A}=c \mid N_{k}^{A}=n, N_{k}^{T}=m, N_{k}^{R}=r\right] P\left[N_{k}^{A B}=n-j+i-c \mid N_{k}^{B}=i\right]\right.}_{(e)} \\
& \underbrace{P\left[N_{k}^{A l}=j-i+c \mid N_{k}^{I}=N-i-m-r\right]}_{(f)}
\end{aligned}
$$

$P\left(c_{2}, n, K-m-r\right)=$

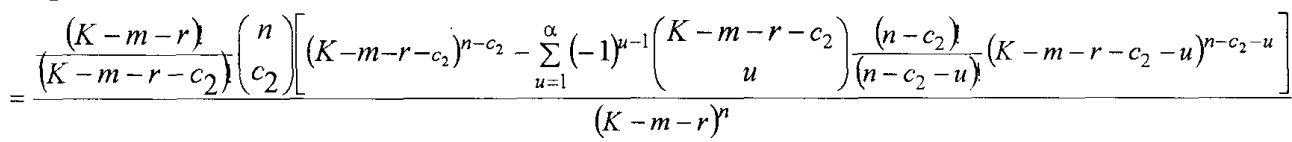

$$
\begin{aligned}
& \alpha=\left\{\begin{array}{cc}
n-c_{2} & \text { if } K-m-r \geq n \\
K-m-r-c_{2}-1 & \text { if } n>K-m-r
\end{array}\right.
\end{aligned}
$$


By making use of expressions (3)-(12) we are able to evaluate the state transition probability for the case $K-m-r>0$.

When there are no available codes, $(K-m-r=0)$, possible transitions are slightly different from those presented in figure 1. Particularly, it must be noted that in this case any terminal neither from idle mode nor from backlogged mode will try to acquire a code because the base station is broadcasting all codes as busy. Consequently, all the terminals that receive a new message automatically will go to backlogged mode and they will remain there until code availability is broadcasted by the base station.

By carrying out a similar analysis, it is easy to show that the state transition probability for the case $K$ $m-r=0$ is given by the following expression:

$$
\begin{aligned}
& p_{i m r, j l s}=\left(\begin{array}{c}
N-m-r-i \\
j-i
\end{array}\right) p_{i}^{j-i}\left(1-p_{i}\right)^{N-m-r-j} \\
& \sum_{t=\max (0, r-s)}^{\min (r, m-s+r, l)}\left(\begin{array}{c}
m-s+r-t \\
m-l-s+r
\end{array}\right)\left(\begin{array}{c}
m \\
s-r+t
\end{array}\right)\left(p_{o k}(m) p_{m}\right)^{m-l-s+r} \\
& \left(1-p_{o k}(m) p_{m}-\left(1-p_{o k}(m)\right)\left(1-p_{b}\right)\right)^{-t} \\
& \left(\left(1-p_{o k}(m)\right)\left(1-p_{b}\right)\right)^{s-r+t}\left(\begin{array}{l}
r \\
t
\end{array}\right) p_{b}^{t}\left(1-p_{b}\right)^{r-t}
\end{aligned}
$$

which is valid for $j \geq i$ and $m+r-l-s \geq 0$, being 0 in all other cases. The first condition is related to the fact that when there are not available codes, it is not possible to reduce the number of users in backlogged mode from time slot $k$ to $k+1$, and thus necessarily $j \geq i$. Similarly, it is not possible to increase from slot $k$ to $k+1$ the total number of users that have acquired a code, and consequently $m+r \geq l+s$.

After calculating the state transition probabilities, we can construct the state transition matrix $\mathbf{P}$ and find the steady state probability vector $\pi=\left\{\pi_{\mathrm{i}, \mathrm{m}, \mathrm{r}}\right\}$ by solving the system $\pi=\pi P$ with the condition:

$$
\sum_{r=0}^{K} \sum_{m=0}^{K-r N-m-r} \sum_{i=0}^{N} \pi_{i, m, r}=1
$$

The system throughput can then be calculated as $S=S^{T}+S^{T}, S^{T}$ being the mean number of correctly transmitted packets in the transmission mode and $S^{I}$ being the mean number of successful code acquisitions, defined as:

$$
\begin{gathered}
S^{T}=\sum_{l=0}^{K} l \sum_{i, m, r} \sum_{a=0}^{N-r-m-i} \sum_{n=a}^{i+a}\left(\begin{array}{c}
m \\
l
\end{array}\right)\left(p_{o k}(m+n)\right)^{l}\left(1-p_{o k}(m+n)\right)^{m-l} \\
\left(\begin{array}{c}
i \\
n-a
\end{array}\right) p_{r}^{n-a}\left(1-p_{r}\right)^{-n+a}\left(\begin{array}{c}
N-r-m-i \\
a
\end{array}\right) p_{i}^{a}\left(1-p_{i}\right)^{N-r-m-i-a} \pi_{i, m, r}
\end{gathered}
$$

$$
\begin{aligned}
& S^{I}=\sum_{c=0}^{K} c \sum_{i, m, r} \sum_{a=0}^{N-r-m-i} \sum_{n=a}^{i+a} P\left[N^{S A}=c \mid N^{A}=n, N^{T}=m, N^{R}=r\right] \\
& \left(\begin{array}{c}
i \\
n-a
\end{array}\right) p_{r}^{n-a}\left(1-p_{r}\right)^{j-n+a}\left(\begin{array}{c}
N-r-m-i \\
a
\end{array}\right) p_{i}^{a}\left(1-p_{i}\right)^{N-r-m-i-a} \pi_{i, m, r}
\end{aligned}
$$

with $P\left[N^{S A}=c \mid N^{A}=n, N^{T}=m, N^{R}=r\right]$ given in (10).

In order to validate the model, some simulations have been carried out. Results are depicted in figure 2 , where it can be observed that the model fits well with simulation results.

\section{3.- System performance. Effect of the number of codes $K$.}

One of the most important parameters in the ISMA CDMA system is the total number of codes $K$ that will be available for the different users. Particularly, it is interesting to note that $K$ must be high enough in order to reduce the number of collisions, but, on the other hand, in order to reduce the complexity of receivers, the amount of feedback information, and also to allow the selection of a finite set of codes with good cross-correlation properties, $K$ must be kept as low as possible. So we are interested in finding which is the optimum value for $K$

We must consider the fact that the system is not only limited by collisions but also by multiuser interference. The main point here, then, is that $K$ has a great influence over the probability of collision, while its influence over the interference is less important. Consequently, the gain due to an increase in the value of $K$ is not to be observed whenever the error probability due to interference is much higher than the collision probability. In other words, we can increase $K$ and observe an improvement on system performance until the point where the collision probability becomes lower than the error probability due to multiuser interference. This effect is shown in figure 3 where the throughput of ISMA CDMA is presented for different values of $K$ and for the case when $S_{f}=64$ and $L=640$ bits, which corresponds to one of the specified bit rates for the UTRA proposal [4]. It can be observed that a significant gain in the maximum throughput is reached when employing 20 codes instead of 10 , and also when increasing up to 30 codes. However, no gain is observed when employing a higher number of codes.

In order to better delimit which is this maximum number of codes, figure 4 presents the regions where the collision probability is lower than the error probability due to multiuser interference meaning that the system is interference limited and the opposite case where the system is collision 
limited, for different values of the total offered load (bits/slot) and the number of codes $K$. It is shown that the maximum offered load that keeps the system interference limited increases with $K$. However, beyond a maximum value $K_{\max }$, (approximately 25 codes for the case $L=640$, $S_{f}=64$ ), this maximum offered load remains approximately constant, which means that no gain is obtained by employing a higher number of codes than $K_{\max }$.

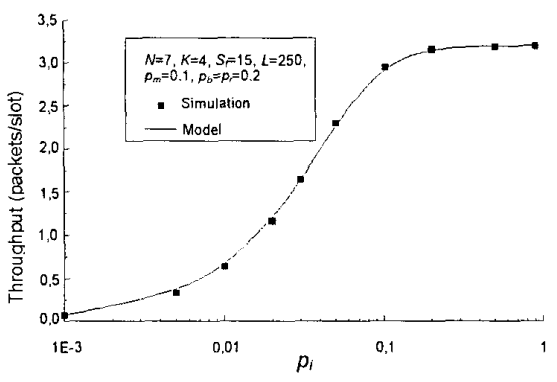

Figure 2 Comparison between simulated and theoretical throughput

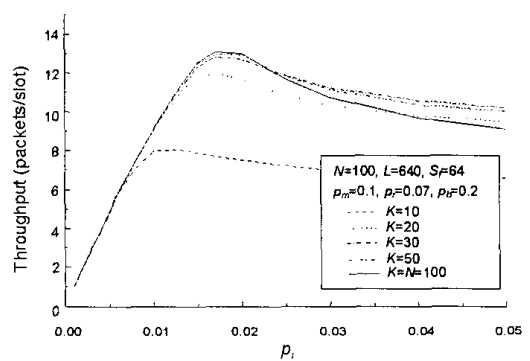

Figure 3 Throughput of ISMA CDMA for different values of the number of codes $K$

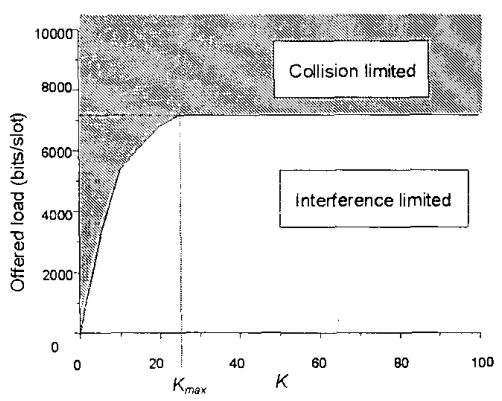

Figure 4 Regions where the system is collision or interference limited for the case $S_{f}=64, L=640$.

In order to try the generalization of the last result, the same process has been carried out for other two bit rates specified in UTRA, specifically $S_{f}=32$, $L=1280$ and $S_{f}=128, L=320$. The results are presented in table $I$. In addition, the maximum throughput $S_{\max }$ that could be reached in the absence of collisions for each one of the specified bit rates is also presented. This throughput is obtained by maximizing the number of successful transmitted packets when there are $x$ simultaneous transmissions, expression given by:

$S=x\left(1-Q\left(\sqrt{\frac{3 S_{f}}{x-1}}\right)\right)^{L}$

The general conclusion that can be extracted from table I is that approximately the optimum number of codes to be used for a given bit rate is around twice the maximum throughput in packets/slot that would be obtained in the absence of collisions.

\begin{tabular}{|c|c|c|c|}
\hline $\boldsymbol{S}_{f}$ & $\boldsymbol{L}$ (bits) & $\boldsymbol{K}_{\max }$ & $\boldsymbol{S}_{\max }$ \\
\hline 128 & 320 & 60 & 30.68 \\
\hline 64 & 640 & 25 & 14.22 \\
\hline 32 & 1280 & 15 & 6.93 \\
\hline
\end{tabular}

Table I Maximum number of codes for three different bit rates

\section{4.- Conclusions}

In this paper, an analytical model for a ISMA CDMA packet data network has been presented. Additionally, performance analysis has been carried out for different values of the number of codes in the system $K$. It has been found that for a given bit rate defined by the spreading factor and the packet length, the optimal number of codes to employ is around twice the maximum throughput in packets/slot that could be obtained in the absence of collisions.

\section{REFERENCES}

[1] K. Zdunek, D.R. Ucci, J. L.LoCicero, "Packet Radio Performance of Inhibit Sense Multiple Access with Capture", IEEE Trans. On Comm. Vol $45, n^{\circ} 2$, February 1997, pp $164-167$

[2] A. Chockalingam, W.Xu, L.B. Milstein, "Performance of a Multi-Channel Packet CDMA Protocol in a Fading Environment", Proc. VTC'97, Phoenix, Arizona, USA, 1997.

[3] M. B. Pursley, "Performance Evaluation for Phase-Coded Spread - Spectrum Multiple - Access Communicaton - Part I: System Analysis", IEEE Trans. On Commun., Vol. Com 25, $\mathrm{n}^{\circ} 8$, August, 1977, pp 795-799.

[4] E.Dahlman, P. Beming, J. Knutsson, F. Ovesjö, M. Persson, C. Roobol, "WCDMA - The Radio Interface for Future Mobile Multimedia Communications", IEEE Trans. On. Vehic. Techn., vol 47, n²4, November, 1998, pp 1105-1118

[5] O. Sallent, R.Agusti, "System Design Issues in a Code-Restricted Packet Data DS-CDMA Network", Proc. ICT'98, Porto Carras, Greece, 1998 